\title{
Tackling critical parameters in Metazoan meta-barcoding experiments: a preliminary study based on coxI DNA barcode
} \author{
, Donatella Cesaroni ${ }^{4}$, Maurizio Casiraghi ${ }^{3}$, Saverio Vicario ${ }^{8}$ \\ ${ }^{1}$ Istituto di Biomembrane, Bioenergetica e Biotecnologie Molecolari - Consiglio Nazionale delle Ricerche, Bari, Italy \\ 2 Dipartimento di Biologia, Università degli studi di Bari 'Aldo Moro', Bari, Italy \\ 3 Dipartimento di Biotecnologie e Bioscienze - Zooplantlab, Università degli studi di Milano Bicocca, Milan, Italy \\ 4 Dipartimento di Biologia, Università di Roma Tor Vergata, Rome, Italy \\ 5 Istituto di Tecnologie Biomediche - Consiglio Nazionale delle Ricerche, Bari, Italy \\ 6 Istituto di Biologia Agroambientale e Forestale - Consiglio Nazionale delle Ricerche, Rome, Italy \\ 7 Dipartimento di Bioscienze, Biotecnologie e Biofarmaceutica, Università degli Studi di Bari 'Aldo Moro', Bari, Italy \\ 8 Istituto sull'Inquinamento Atmosferico - Consiglio Nazionale delle Ricerche, Bari, Italy \\ Corresponding Author: Bachir Balech \\ Email address: balechbachir@gmail.com
}

Bachir Balech Corresp., 1,2 , Anna Sandionigi ${ }^{3}$, Caterina Manzari ${ }^{1}$, Emiliano Trucchi ${ }^{4}$, Apollonia Tullo ${ }^{1,5}$, Flavio Licciulli $^{5}$, Giorgio Grillo ${ }^{5}$, Elisabetta Sbisà ${ }^{5}$, Stefano De Felici ${ }^{4,6}$, Cecilia Saccone ${ }^{7}$, Anna Maria D’Erchia

Nowadays DNA meta-barcoding is a powerful instrument capable of quickly discovering the biodiversity of an environmental sample by integrating the DNA barcoding approach with High Throughput Sequencing technologies. It mainly consists of the parallel reading of informative genomic fragment/s able to discriminate living entities. Although this approach has been widely studied, it still needs optimization in some necessary steps requested in its advanced accomplishment. A fundamental element regards the standardization of bioinformatic analyses pipelines. The aim of the present study was to underline a number of critical parameters of laboratory material preparation and taxonomic assignment pipelines in DNA meta-barcoding experiments using the cytochrome oxidase subunit-I (coxl) barcode region, known as a suitable molecular marker for animal species identification. We compared nine taxonomic assignment pipelines, of which a custom inhouse method, based on Hidden Markov Models. Moreover, we evaluated the potential influence of universal primers amplification bias in qPCR, as well as the correlation between GC content with taxonomic assignment results. The pipelines were tested on a community of known terrestrial invertebrates collected by pitfall traps from a chestnut forest in Italy. Although the present analysis was not exhaustive and needs additional investigation, our results suggest some potential improvements in laboratory materials preparation and the introduction of additional parameters in taxonomic assignment pipelines. These include the correct setup of OTU clustering threshold, the calibration of GC content affecting sequencing quality and taxonomic classification, as well as the evaluation of PCR primers amplification bias on the final biodiversity pattern. Thus, careful 
attention and further validation/optimization of the above-mentioned variables would be required in a DNA meta-barcoding experimental routine. 


\section{Tackling critical parameters in metazoan meta-barcoding 2 experiments: a preliminary study based on coxI DNA 3 barcode}

4 Bachir Balech ${ }^{1,2}$, Anna Sandionigi ${ }^{3}$, Caterina Manzari ${ }^{1}$, Emiliano Trucchi ${ }^{4}$, Apollonia Tullo ${ }^{1,5}$, Flavio

5 Licciulli $^{5}$, Giorgio Grillo ${ }^{5}$, Elisabetta Sbisà ${ }^{5}$, Stefano De Felici ${ }^{4,6}$, Cecilia Saccone ${ }^{7}$, Anna Maria

6 D’Erchia $^{7}$, Donatella Cesaroni ${ }^{4}$, Maurizio Casiraghi ${ }^{3}$, Saverio Vicario ${ }^{8}$

$7{ }^{1}$ Istituto di Biomembrane, Bioenergetica e Biotecnologie Molecolari - Consiglio Nazionale delle

8 Ricerche, Bari, Italy

$9 \quad 2$ Dipartimento di Biologia, Università degli Studi di Bari ‘Aldo Moro', Bari, Italy

$10{ }^{3}$ Dipartimento di Biotecnologie e Bioscienze - Zooplantlab, Università degli studi di Milano Bicocca,

11 Milan, Italy

$12{ }^{4}$ Dipartimento di Biologia, Università di Roma Tor Vergata, Rome, Italy

$13{ }^{5}$ Istituto di Tecnologie Biomediche - Consiglio Nazionale delle Ricerche, Bari, Italy

$14{ }^{6}$ Istituto di Biologia Agroambientale e Forestale - Consiglio Nazionale delle Ricerche, Monterotondo

15 Stazione- Rome, Italy

$16{ }^{7}$ Dipartimento di Bioscienze, Biotecnologie e Biofarmaceutica, Università degli Studi di Bari 'Aldo

17 Moro', Bari, Italy

$18{ }^{8}$ Istituto sull'Inquinamento Atmosferico - Consiglio Nazionale delle Ricerche, Bari, Italy

Corresponding Author:

21 Bachir Balech ${ }^{1,2}$

22 Via Amendola 165/A, Bari, 70126, Italy

23 Email address: balechbachir@gmail.com 
Abstract

Nowadays DNA meta-barcoding is a powerful instrument capable of quickly discovering the biodiversity of an environmental sample by integrating the DNA barcoding approach with High Throughput Sequencing technologies. It mainly consists of the parallel reading of informative genomic fragment/s able to discriminate living entities. Although this approach has been widely studied, it still needs optimization in some necessary steps requested in its advanced accomplishment. A fundamental element regards the standardization of bioinformatic analyses pipelines. The aim of the present study was to underline a number of critical parameters of laboratory material preparation and taxonomic assignment pipelines in DNA meta-barcoding experiments using the cytochrome oxidase subunit-I (coxI) barcode region, known as a suitable molecular marker for animal species identification. We compared nine taxonomic assignment pipelines, of which a custom in-house method, based on Hidden Markov Models. Moreover, we evaluated the potential influence of universal primers amplification bias in qPCR, as well as the correlation between GC content with taxonomic assignment results. The pipelines were tested on a community of known terrestrial invertebrates collected by pitfall traps from a chestnut forest in Italy. Although the present analysis was not exhaustive and needs additional investigation, our results suggest some potential improvements in laboratory materials preparation and the introduction of additional parameters in taxonomic assignment pipelines. These include the correct setup of OTU clustering threshold, the calibration of GC content affecting sequencing quality and taxonomic classification, as well as the evaluation of PCR primers amplification bias 
54 on the final biodiversity pattern. Thus, careful attention and further validation/optimization of the 55 above-mentioned variables would be required in a DNA meta-barcoding experimental routine.

56

57

58

59

60

61

62

63

64

\section{Introduction}

The introduction of DNA barcoding (Hebert et al. 2003) shed new light on the identification process of many life forms on earth leading to a wider comprehension of many ecosystems both aquatic and terrestrial (Aylagas et al. 2014; Comtet et al. 2015). DNA barcoding was originally designed to identify single organisms, but scientific progresses have adapted it to a new but similar technique called "DNA meta-barcoding". This mainly consists of the parallel reading of informative genomic fragment/s able to discriminate living entities (Taberlet et al. 2012). DNA barcoding and meta-barcoding share the common process in flagging a specific DNA sequence to a taxonomic name, hopefully at species level (Hajibabaei 2012). However, compared to DNA barcoding, the meta-barcoding approach requires a reduced sampling effort to collectively characterize the inhabitant organisms of a given environment (Coissac et al. 2012; De Barba et al. 2014). Supported by the High Throughput Sequencing (HTS) technologies (Bik et al. 2012; Shokralla et al. 2012), DNA meta-barcoding is revolutionizing ecological studies by expanding the information on ecosystem biodiversity (Kajtoch 2014). This innovative tool is also widely used for monitoring purposes such as invasive species control (Comtet et al. 2015). Moreover, its ability to identify fungi (Bellemain et al. 2013), plants (Quemere et al. 2013), chromista (Nanjappa et al. 2014), bacteria (Sogin et al. 2006) and metazoans (Leray \& Knowlton 2015) from the same sample or ecological area is of great importance to understand natural connections among these life forms and consequently to plan ecosystem monitoring and/or biodiversity conservation programs (Ji et al. 2013).

Many molecular markers are currently used to characterize species or taxa from all life domains, for instance, ITS (Internal Transcribed Spacer) targets fungi (Geml et al. 2014; Op De Beeck et al. 2014), 16S ribosomal RNA is employed in bacterial identification (Fantini et al. 2015) and $\operatorname{trnL}$ (UAA) intron (Srivathsan et al. 2015) or rbcL-matK (large subunit of RUBISCO - Maturase $\mathrm{K})$ (Xu et al. 2015) are accustomed to plants. Besides, the use of the cytochrome oxidase subunit-I ( $\operatorname{cox} I)$ molecular marker in animals' identification is increasingly gaining greater importance in DNA barcoding and meta-barcoding studies (i.e. (Mollot et al. 2014)). Thanks to 
85

86

87

HTS technologies, the above-mentioned molecular markers can be used simultaneously in order to target a broad range of life forms from the same experimental sample (e.g. (Gibson et al. 2014; Zhan et al. 2014). However, this massive and integrative approach is still method sensitive, which requires different levels of standardization and optimization in its implementation steps (Cristescu 2014), including both laboratory materials and techniques and bioinformatics analyses. Once all those steps are standardized, it becomes possible to compare different experiments carried out by a meta-barcoding approach. For instance, the use of efficient and lowbiased universal PCR primer pairs is crucial to get a successful DNA amplification of the majority of organisms present in the environmental sample under investigation. Recent studies highlighted the influence of universal primers amplification bias on the accuracy of species diversity reconstruction and their relative abundances (Geisen et al. 2015; Pawluczyk et al. 2015; Pinol et al. 2014). Sequencing chemistries prone to errors caused by GC content are also of fundamental importance as they can alter the true picture of organismal composition (Abnizova et al. 2012). Additional variables, which may influence the quality of meta-barcoding sequences processing, include the availability of well-represented reference databases (Cowart et al. 2015) and the compatibility of taxonomic assignment tools with the DNA information of the molecular marker in use (Balint et al. 2014). Consequently, to reach the relevant conclusions from a metabarcoding assay, bioinformatic analyses pipelines would require some standardization taking into account the aforementioned variables. A typical pipeline in DNA meta-barcoding data analysis consists of three parts: (1) sequence denoising or quality filtering; (2) operational taxonomic units (OTU) picking and (3) taxonomic assignment at species and/or higher levels. Once the denoising protocol has been defined (Edgar et al. 2011; Ficetola et al. 2015; Quince et al. 2011; Schloss et al. 2011), two critical and dependent parameters can potentially influence the quality of taxonomic assignment, namely OTU clustering (Chen et al. 2013; Edgar 2013) and classification (Bacci et al. 2015) thresholds. The clustering threshold is directly correlated to the intra-specific distance and at the same time to the sequencing error rate. This can guide significantly the accuracy and precision of the subsequent taxonomic identification, influenced by the correct classification threshold, and the relative abundance assigned to each classified taxon.

Here we present a preliminary study aimed to highlight a set of critical parameters of taxonomic assignment pipelines used in coxI DNA meta-barcoding analyses, namely OTU clustering, GC 
116 content and PCR primers amplification bias. For that, we compared nine taxonomic assignment

117 pipelines, of which a custom in-house one based on Hidden Markov Models (HMM). The

118 taxonomic assignments obtained by the best pipeline were then evaluated for their potential

119 influence by universal primers amplification bias (using qPCR) and by GC content. The pipelines

120 were tested on a community of known terrestrial invertebrates, taxonomically classified based on

121 their morphology, collected by pitfall traps from a chestnut forest in Italy.

122

123

124

125

126

127

128

129

130

131

132

133

134

135

136

137

138

139

140

141

142

143

144

145

\section{Materials and Methods}

\section{Samples description}

The sampling unit consisted of two samples containing soil litter macrofauna, called MPE4 and MPE5. MPE5 is the result of pooling the content of five pitfall traps, placed in circle at $10 \mathrm{~m}$ of distance each, in a chestnut forest located in central Italy (province of Rome). All collected organisms belonging to the Carabidae family (order: Coleoptera) were morphologically classified at species level, while the others were labeled with their order (whenever possible) or class rank names. The biomass of each organism was measured singularly for further statistical analysis. MPE4 is an ad-hoc sample composed from the equal biomass content of all the organisms present in MPE5, using the same weight of the lightest organism (see Table. 1 for organisms' names and their corresponding biomass).

\section{DNA extraction and amplification of coxI barcode}

Each sample, MPE4 and MPE5, was homogenized separately and total genomic DNA was extracted using the DNeasy Blood and Tissue (Qiagen) commercial kit. In addition, total DNA was extracted from the Carabidae species individually. The coxI DNA barcode was amplified from all DNA extracts using the universal primer pairs (Folmer et al. 1994): forward-LCO1490 (5'-GGTCAACAAATCATAAAGATATTGG-3'), and reverse-HCO2198 (5'-

TAAACTTCAGGGTGACCAAAAAATCA-3'). PCR reactions were carried out in $50 \mu 1$ reaction volumes containing: $1.5 \mathrm{mM} \mathrm{MgCl} 2,250 \mathrm{nM}$ of each primer, $200 \mu \mathrm{M}$ of each dNTP, $1 \mathrm{x}$ of Phusion HF Buffer, 1U of Phusion DNA polymerase (M0530S, NEB) and $2 \mu$ of DNA extracts, using a thermocycling profile of one cycle of $60 \mathrm{~s}$ at $94^{\circ} \mathrm{C}$, five cycles of $60 \mathrm{~s}$ at $94^{\circ} \mathrm{C}$, $90 \mathrm{~s}$ at $45^{\circ} \mathrm{C}$, and $90 \mathrm{~s}$ at $72^{\circ} \mathrm{C}$, followed by 35 cycles of $60 \mathrm{~s}$ at $94^{\circ} \mathrm{C}, 90 \mathrm{~s}$ at $50^{\circ} \mathrm{C}$, and $60 \mathrm{~s}$ at $72^{\circ} \mathrm{C}$, with a final step of 5 min at $72^{\circ} \mathrm{C}$. PCR products along with $100 \mathrm{bp}$ DNA Ladder 
146 (Fermentas, Life Sciences) were visualized on a 1\% agarose gel stained with $0.005 \%$ of ethidium

147 bromide. PCR products were subsequently gel purified using QIAquick Gel Extraction Kit

148 (Qiagen).

149 Table 1.

150 Sequencing

151 The coxI barcode region was sequenced singularly for each Carabidae organism by Sanger

152 method. These sequences were then used as controls in the following experimental and analysis

153 steps. Furthermore, MPE4 and MPE5 samples were prepared for pyrosequencing adapting the

154 sequencing library preparation protocol described by Calabrese et al. (2013). The libraries were

155 then deposited on 2/8-lane PicoTiterPlate (PTP) wells (Roche/454) and sequenced in both

156 directions on GS FLX Titanium pyrosequencing platform.

157

158

\section{Bioinformatics analysis}

159 The bioinformatics analysis was mainly divided into three steps: (i) sequence reads filtering and 160 denoising, (ii) OTU picking and (iii) taxonomic assignment. In this study, we compared nine 161 pipelines (Fig. 1), of which a custom one based on Hidden Markov Models. For the sake of 162 simplicity, in the rest of the manuscript we will call the custom pipeline HSTA (Hidden States 163 Taxa Assign). However, we followed a standard procedure in the first step and we used several 164 OTU picking algorithms with progressive thresholds and different taxonomic assigners in the 165 second and third steps respectively. The tested pipelines are based on (1) Usearch, Uclust and 166 Blast as OTU picking methods (thresholds: 100, 97, 95, 90\%) in combination with RDP and 167 BLAST classifiers (all available in Qiime (Caporaso et al. 2010)); (2) Usearch-Ref, Uclust-Ref 168 (thresholds: 100, 97, 95, 90\%) (Edgar 2013) and HSTA as direct taxonomic assigners without 169 calling OTUs.

170 Sequence reads filtering and denoising

171 We performed a standard read rejecting and trimming procedure, using GS Run Processor V2.4

172 (Roche 454 Life Sciences software package), with the parameters suggested by the 454 platform 173 manufacturer. Due to amplicons ligation and subsequent nebulization steps carried out for 174 sequencing library preparation (see Calabrese et al. 2013), we executed a primer search analysis 
175 (Supplemental S1) generating forward and reverse reads data sets, which were processed 176 separately in the subsequent analyses. Pyrosequencing and PCR noises were removed by 177 invoking PyroNoise and SeqNoise algorithms respectively, available from AmpliconNoise 178 package (Quince et al. 2011), with their default parameters. Denoised sequences were then 179 submitted to chimera detection and removal using UCHIME (de novo method) (Edgar et al. 180 2011). At this point, we obtained two denoised data sets per sample corresponding to the 5' and 181 3' ends of coxI barcode region.

Figure 1.

We applied the de-novo OTU picking method of Usearch, Uclust and Blast algorithms, available in Qiime (Caporaso et al. 2010), at four different similarity thresholds: 100, 97, 95 and 90\%. OTUs were taxonomically classified by RDP and BLAST classifiers with their default parameters. In addition, without calling OTUs, a direct taxonomic assignment was carried out by Usearch-Ref and Uclust-Ref (Edgar 2013) at four different similarity thresholds (100, 97, 95 and $90 \%$ ) and by HSTA. Note that the same coxI reference database was used for all tested pipelines (see below for additional information). Given that HSTA was assembled as a custom pipeline, in the following we provide a description of its main steps, namely (a) the choice of reference sequences, (b) reference Hidden Markov Models (HMM) profiles building and (c) taxonomic classification method:

196

(a) Choice of reference sequences

197

Several sources of coxI reference sequences were taken into account in building a coxI reference database. First of all, a local database (LocalDB) was generated from the coxI DNA barcode sequences, produced in this study, of 114 Carabidae individuals belonging to seven different species classified morphologically at species level (species names are listed in Table 1). On the other hand, public coxI sequences were obtained by blastx (Altschul et al. 1990) using the denoised sequences as queries against BOLD (http://boldsystems.org/) and GenBank (NR-NCBI) databases. Blastx outputs were parsed by 
204 rank name (NCBI taxonomy). All 10 matched references per query were filtered to select only the entries

205 which had the same taxonomical order of the best-hit match.

206

207

208

(b) Reference HMM profiles building

209

Amino acid reference sequences selected in the previous step (belonging to each order and species ranks) were multiple aligned using Muscle 3.8 .31 (Edgar 2004). Multiple protein alignments were used to generate the relevant nucleotide ones, denoted as back-alignment, as described by Balech et al (2015). Hidden Markov Model (HMM) profiles were then built from the nucleotide alignments using hmmbuild

(c) Taxonomic classification method

215

216

217

218

219

220

221

222

223

224

225

226

227

228

229

230

231

232

233

234 (HMMer 3.0) with its default parameters (Finn et al. 2011).

Denoised sequences were assigned to one of the nucleotide HMM profiles by executing hmmscan (HMMer 3.0) with its default parameters. The outputs were then parsed using a Python 2.7 script which classifies the assigned sequence in three categories:

- Unclassified assignment: sequence-profile match outputs an e-value higher than hmmscan default one.

- Good assignment: the best match bit score passes the threshold of Bayes Factor $(B F)$ (Eq. 1), set to 3.0. $B F$ is computed by subtracting the best bit score $\left(S_{i=1}\right)$ from the natural logarithm of the sum of all $\left(S_{i=2-n}\right)$ remaining exponential bit scores.

$$
\ln (B F)=S_{i=1}-\ln \left(\sum_{i=2}^{n} \exp \left(S_{i}\right)\right)
$$

- Ambiguous assignment: sequence-profile hit passes hmmscan threshold but does not pass $B F$ test.

\section{Taxonomic assignment pipelines comparison}

To validate how well the tested pipelines fit, we calculated the ratio of the detected taxa by each tested pipeline against the expected ones. Thus, the taxonomically classified reads were searched for the expected Carabidae species and order level taxa listed in Table 1. In addition, Pearson's correlations were calculated between read abundances relative to each taxon and its corresponding biomass.

\section{GC content assessment}

GC content assessment was conducted to infer its potential relationship with sequencing errors and consequently its influence on the denoising and taxonomic assignment processes behavior. 
235 We therefore, hypothesized that the denoising procedure should be calibrated based on GC

236 content effect, which potentially influences the sequencing reaction. To test this hypothesis, we

237 assessed the contribution of the expected biological variation and the GC content (both present in

238 the reference sequences) on denoised reads variation taking into consideration the sequences

239 assigned to Coleoptera order (the taxonomic group with most abundant assigned reads and 240 reference sequences). We used a linear model "Eq. (2)" to predict the change in diversity across 241 sites.

$$
\text { Dreads } \sim \text { Dref }+ \text { GCref }
$$

243 In (Eq. 2) Dreads and Dref are the exponential entropy per site in reads assigned to Coleoptera and the reference sequences respectively, while GCref is the mean GC percentage over a 100bp window in the reference sequences. We used the exponential entropy per site following Jost (2006) as an example of the diversity index for categorical variable. The model was estimated separately for the 5' coxI barcode, taking the first $400 \mathrm{bp}$ of the Coleoptera nucleotide multiple alignments (reference and assigned reads), and the 3 ' one starting from the $200^{\text {th }}$ site until the end.

\section{Species level assignment evaluation with qPCR}

To explain the correlation observed between the abundance of assigned sequences and taxon biomass at species level, we adopted a qPCR approach to track the number of coxI copies in two different Carabidae species across all experimental steps. We therefore compared the ratio of coxI copies, the biomass and the assigned sequence abundance obtained from HSTA (the pipeline that detected the highest number of known species). Accordingly, two specific primer pairs were designed for CC1 (Carabus (Tomocarabus) convexus dilatatus; forward: 5'ATTCTGGCTCCTACCTCCG-3', reverse: 5'-CTGCCCCTAAAATTGATGAG-3) and LL1 (Laemostenus latialis; forward: 5'- TACGATCTACAGGAATAACC -3', reverse: 5'AGCAGGGTCAAAAAAGGAT -3’). qPCR reactions, using SYBR Green as a fluorescent reporter, were conducted in $50 \mu \mathrm{l}$ reaction volumes containing $2 \mu \mathrm{l}$ of DNA template, $300 \mathrm{nM}$ of each primer and $22.5 \mu$ of RealMasterMix SYBR ROX 2.5x (5 Prime): 1U Taq DNA Polymerase, $4 \mathrm{mM}$ Magnesium Acetate, $0.4 \mathrm{mM}$ of each dNTP, using a thermocycling profile of one cycle of $2 \mathrm{~min}$ at $94^{\circ} \mathrm{C}, 45$ cycles of $30 \mathrm{sec}$ at $94^{\circ} \mathrm{C}, 30 \mathrm{sec}$ at $60^{\circ} \mathrm{C}$ and $40 \mathrm{sec}$ at $68^{\circ} \mathrm{C}$. The 
265

266

267

268

269

270

271

272

273

274

275

276

277

278

279

280

281

282

283

284

285

286

287

288

289

290

291

292

DNA templates consisted of MPE4 and MPE5 DNA extracts, their PCR products obtained from the amplification with Folmer primer set and the DNA yielded from libraries preparation for 454 sequencing. The reactions were performed on a 7900 HT Fast Real-time PCR system (Applied Biosystem). A total of 18 replicates per primer set and per template were performed (six replicates per template over three different qPCR plates) and the resulting data was analyzed by MAK2 (Boggy \& Woolf 2010).

\section{Results}

\section{Sequencing yield and taxonomic assignment}

A total of 160166 passed filter reads were obtained for both samples (MPE4: 67672, MPE5: 92494). Following the primer search analysis (Supplemental S1) and subsequent denoising, 2595 denoised reads were obtained for MPE5 (650 for the 5' and 1945 for the 3') and 5368 for MPE4 (1271 for the 5' and 4097 for the 3'). In HSTA and reference-based assignments (Usearch-Ref and Uclust-Ref), we used denoised reads as a direct input for down streaming analysis, while we applied an OTU picking step for the other methods (see materials and methods).

Comparing the taxonomic assignment results obtained in at order level, at 5 ' end (Fig. 2A) HSTA could detect seven out of 11 expected orders followed by five orders identified by Usearch+BLAST (OTU picking method+Classifier) at 97, 95 and 90\% and Uclust+BLAST at 95 and $90 \%$ OTU picking thresholds. As for the 3', five orders were found by Usearch+BLAST and Uclust+BLAST at 100\% OTU picking threshold followed by HSTA detecting only three (Fig. 2B).

Regarding the assignment at species level (Fig. 3), out of seven expected species, HSTA could classify all of the searched species in both the 5' and 3' datasets. Furthermore, at 5', Blast+RDP (OTU picking method+Classifier) showed stable behavior as it detected five species at all OTU picking thresholds. The same result was obtained for Usearch+BLAST at 97 and $90 \%$ and Uclust+BLAST at 95 and 90\% OTU picking threshold (Fig. 3A). At the 3' end (Fig. 3B), the OTU picking methods Uclust and Blast with RDP classifier appear to be consistent over all similarity thresholds classifying four out of seven expected species. Moreover, it is important to 
293 note that reference-based algorithms (Usearch-Ref and Uclust-Ref) were able to uncover a lower

294 number of expected taxa than classifiers did.

295 Pearson's correlation values (calculated only when more than one taxon was classified) of the

296 assigned reads abundance against the corresponding taxa biomass demonstrated linear behavior

297 with the qualitative results described above, confirming the accuracy of almost all tested

298 taxonomic assignment pipelines. Overall, these correlations were higher at order level

299 assignments (greater or equal to 0.98) compared to species ones (ranged from 0.86 to 0.92). The

300 only two exceptions, at species level, showing respectively negative correlations of -0.22 and -

3010.79 at $100 \%$ similarity threshold were Blast+RDP and Uclust-Ref.

\section{Figure 2.}

303 Figure 3.

304 GC content assessment

305 GC content assessment was conducted separately for the 5' coxI barcode, taking the first 400bp

306 of the Coleoptera nucleotide multiple alignment, and the 3 ' one starting from the $200^{\text {th }}$ site until

307 the end. As expected, the linear model results (Table 2) showed that the diversity index

308 (exponential value of entropy) of the assigned sequences is explained by that of reference

309 sequences. In addition, the variability of diversity value in denoised sequences weighed by GC

310 content was statistically significant at both the 5' and 3' coxI barcode. Nevertheless, the sum of

311 squares values pointed out that this significant variability due to GC content is larger at the 3'

312 (38 corresponding to $17 \%$ of explained variance) than that at the 5 ' (4.5 equivalent to $2.4 \%$ ).

313 This indicates some shortcomings in denoising protocol given by its potential inability to remove

314 the excess of errors due to GC content.

Table 2.

317

318

Species level assignment validation with qPCR

319

To perform a quantitative validation of organisms biomass effect on taxonomic assignments, we chose two organisms, CC1 and LL1 (Table 1), belonging to the Carabidae family and classified morphologically at species level. We calculated the ratio CC1/LL1 of biomass and that of read 
322 abundance obtained from HSTA (the pipeline that detected the highest number of known

323 species) (Table 3) in both MPE4 and MPE5 samples. At equal biomass (MPE4) CC1 had almost

324 an average of $1.6\left(=\left(1.83\right.\right.$ at $5^{\prime}+1.29$ at $\left.\left.3^{\prime}\right) / 2\right)$ greater assigned reads than LL1, while at 53

325 biomass ratio (in MPE5) read abundance increased significantly to approximately 167 times

$326\left(=\left(231.35\right.\right.$ at $5^{\prime}+103.55$ at $\left.\left.3^{\prime}\right) / 2\right)$ of CC1 over LL1. Moreover, the ratio recorded in DNA

327 extracts appears to be altered when compared to that of PCR products, as it decreased in MPE5

328 (approximately 620) and increased in MPE4 (approximately 164). This emphasizes a potential

329 amplification bias in both samples that is however more manageable in MPE4, where the

330 difference between the initial biomass content and the final read abundance ratios is almost

331 negligible.

332

333

Table 3.

334

335

\section{Discussion}

336 Taxonomic profiling based on HTS technologies of species discriminant loci has been and is still being widely used in numerous microbial and invertebrates biodiversity studies where it has showed its feasibility and accuracy in monitoring species of ecological and/or clinical relevance (Brulc et al. 2009; Cristescu 2014; Fonseca et al. 2014; Fonseca et al. 2010; Singh et al. 2009). Considering two control samples of known metazoan organisms, we addressed some of the parameters potentially influencing the outcomes of a coxI meta-barcoding experiment. It can be argued that we did not perform a massive validation on a wide range of samples and on other molecular barcode markers except coxI. However, as a preliminary study the taxonomic assignment pipelines showed promising results in classifying the known organisms already taxonomically classified based on their morphology and their coxI barcode region (sequenced singularly by Sanger method). We therefore reported, according to this specific experiment, some areas of improvement in both laboratory materials preparation and taxonomic assignment methods.

Based on our taxonomic assignments results, the use of a classifier appear to be essential in coxI meta-barcoding as the direct call of the known organisms by reference-aware algorithms (i.e. Userach-Ref and Uclust-Ref) could not satisfy the same outcome as classifiers did. Combining 
352 the results observed for the assignment at order level (Fig. 2), the use of OTU picking methods,

353 Usearch and Uclust, with BLAST classifier showed consistent and similar behavior at both the 5' 354 and 3 ' ends $(0.45 \%$ of detected orders $)$. However, the best results of these pipelines were 355 obtained using different OTU clustering thresholds (97, 95, 90\% at 5' and 100\% at 3'). This 356 underlines the importance of this parameter in taxonomic assignment routine and suggests its 357 calibration according to an internal control sample or a mock community with similar taxonomic 358 composition of the true samples.

359 Considering the assignment at species level (Fig. 3), the HSTA assigner, based on Hidden 360 Markov Models, showed promising results as it could detect the highest number of the searched 361 known organisms at both the 5' and 3' ends. Although this pipeline is only at a prototype status 362 and needs additional validations and improvements, its strength resides in the absence of an OTU clustering step and in the possibility to extend it to a phylogenetic assignment framework (e.g. pplacer (Matsen et al. 2010), RaxML (Stamatakis 2014)), as HMM profiles are built from multiple sequence alignments. Alternatively, it is important to mention that Blast+RDP (OTU picking method+Classifier) also showed stable classification behavior at both coxI ends as they detected five species $(0.71 \%$ of the expected species) independently from OTU picking thresholds. The differences in accuracy among the tested pipelines while varying the taxonomic level (order or species) would be due to the characteristics of coxI as molecular marker. It is worth mentioning that the majority of the tested methods were specifically designed to analyze 16S rRNA meta-barcoding data (Caporaso et al. 2010). Similar observations were also reported by Balint et al (2014) for the Internal Transcribed Spacer (ITS) analysis pipelines, where the correct taxonomic assignment was influenced by the used tools.

374 Concerning GC content we investigated its effect within Coleoptera reference data set, being the

375 biggest data set used in this study and at the same time, it contains the organisms present in our 376 local reference database (see materials and methods OTU picking and Taxonomic Assignment 377 section). GC content is known to influence the quality of sequence reads in both pyrosequencing 378 (Hoff 2009) and Illumina sequencing (Abnizova et al. 2012). In the present work, we clarified 379 this impact on taxonomic assignment pipelines even after applying a standard denoising 380 protocol. In fact, the low correlation between biomass content and the corresponding assigned 381 reads abundance per taxon at species level and the failure to detect several orders, mainly at the 
382 3', in all tested pipelines would be related to GC content. Indeed, the linear regression model 383 results (Table 2) showed that the variability of assigned reads, higher at 3', was explained by GC 384 content effect. These results highlights the importance of this parameter and proposes its introduction in denoising or quality filtering algorithms of meta-barcoding data to improve taxonomic assignment accuracy.

387

388

389

390

391

392

393

394

395

396

397

398

399

400

401

402

403

404

405

406

407

408

409

410

411

Another important aspect not to be ignored in similar environmental DNA sequencing (i.e. eDNA) is the amplification bias of universal primer set used in PCR preparation step. Many studies have illustrated the effect of amplification bias and primers conservation on sequencing yield and therefore on the assigned taxa abundances (Deagle et al. 2014; Op De Beeck et al. 2014; Pawluczyk et al. 2015), as well as the influence of biomass on the correct biodiversity estimation through DNA meta-barcoding experiments (Thomas 2015). Our investigation in this context using a community of known invertebrates is in line with what is stated above. qPCR results demonstrated a considerable fluctuation of species quantity ratio across all experimental steps in MPE5 sample. The same analysis performed on MPE4, a biomass equalized organismal content, provided a more conserved quantity ratios of those two species (Table 3). This emphasizes a potential limit related to the quantitative prospective of coxI DNA meta-barcoding in natural samples (Pinol et al. 2014) and suggests, whenever possible, sample manipulation including biomass equilibration prior to sequencing.

In conclusion, we observe a rapid and ongoing increase in the use of coxI meta-barcoding assays to study molecular biodiversity. However, the taxonomic assignment pipelines remains challenging. In this preliminary study, we highlighted some important parameters, namely the OTU clustering threshold, GC content and PCR primers amplification bias, to be considered in both laboratory protocols and down streaming analyses. We therefore suggest their extensive evaluation and eventually their introduction into in-silico and in-vitro data processing routines. This could be achieved by introducing an internal control sample or a mock community in a sequencing run in order to tackle the above-mentioned parameters and by testing novel assignment methods (i.e. HSTA) on a wide range of samples and on different molecular markers.

\section{Acknowledgements}

We dedicate this article to Cecilia Lanave, long term partner in research of CS, whose coordinated the effort in writing this project. Unfortunately, an abrupt illness did not allow her to 
412 see the end of the project. We would like also to thank Giacinto Donvito, Pasquale Notarangelo

413 and Gaetano Scioscia for providing the access to computational facilities to run AmpliconNoise, 414 Blast and HMM analyses. The authors are also in debt to Michela Barbuto, Annamaria Paluscio 415 and Angela Fortunato for their precious support in sampling and molecular laboratory analyses. 416 Finally, we thank Catriona Isobel Macleod for English revision.

417

418

419

420

421

422

423

424

425

426

427

428

429

430

431

432

433

434

435

436

437

438

439

440

441

442

\section{References}

Abnizova I, Leonard S, Skelly T, Brown A, Jackson D, Gourtovaia M, Qi G, Te Boekhorst R, Faruque N, Lewis K, and Cox T. 2012. Analysis of context-dependent errors for illumina sequencing. $J$ Bioinform Comput Biol 10:1241005. 10.1142/S0219720012410053

Altschul SF, Gish W, Miller W, Myers EW, and Lipman DJ. 1990. Basic local alignment search tool. $J$ Mol Biol 215:403-410. 10.1016/S0022-2836(05)80360-2

Aylagas E, Borja A, and Rodriguez-Ezpeleta N. 2014. Environmental status assessment using DNA metabarcoding: towards a genetics based Marine Biotic Index (gAMBI). PLoS One 9:e90529. 10.1371/journal.pone.0090529

Bacci G, Bani A, Bazzicalupo M, Ceccherini MT, Galardini M, Nannipieri P, Pietramellara G, and Mengoni A. 2015. Evaluation of the Performances of Ribosomal Database Project (RDP) Classifier for Taxonomic Assignment of 16S rRNA Metabarcoding Sequences Generated from Illumina-Solexa NGS. J Genomics 3:36-39. 10.7150/jgen.9204

Balech B, Vicario S, Donvito G, Monaco A, Notarangelo P, and Pesole G. 2015. MSA-PAD: DNA multiple sequence alignment framework based on PFAM accessed domain information. Bioinformatics 31:2571-2573. 10.1093/bioinformatics/btv141

Balint M, Schmidt PA, Sharma R, Thines M, and Schmitt I. 2014. An Illumina metabarcoding pipeline for fungi. Ecol Evol 4:2642-2653. 10.1002/ece3.1107

Bellemain E, Davey ML, Kauserud H, Epp LS, Boessenkool S, Coissac E, Geml J, Edwards M, Willerslev E, Gussarova G, Taberlet P, Haile J, and Brochmann C. 2013. Fungal palaeodiversity revealed using high-throughput metabarcoding of ancient DNA from arctic permafrost. Environ Microbiol 15:1176-1189. 10.1111/1462-2920.12020

Bik HM, Porazinska DL, Creer S, Caporaso JG, Knight R, and Thomas WK. 2012. Sequencing our way towards understanding global eukaryotic biodiversity. Trends Ecol Evol 27:233-243. DOI 10.1016/j.tree.2011.11.010

PeerJ reviewing PDF | (2017:08:20240:2:0:NEW 2 May 2018) 
443 Boggy GJ, and Woolf PJ. 2010. A mechanistic model of PCR for accurate quantification of quantitative

444

445

446

447

448

449

450

451

452

453

454

455

456

457

458

459

460

461

462

463

464

465

466

467

468

469

470

471

472

473

474

475

476
PCR data. PLoS One 5:e12355. 10.1371/journal.pone.0012355

Brulc JM, Antonopoulos DA, Miller ME, Wilson MK, Yannarell AC, Dinsdale EA, Edwards RE, Frank ED, Emerson JB, Wacklin P, Coutinho PM, Henrissat B, Nelson KE, and White BA. 2009. Genecentric metagenomics of the fiber-adherent bovine rumen microbiome reveals forage specific glycoside hydrolases. Proc Natl Acad Sci U S A 106:1948-1953. 10.1073/pnas.0806191105

Calabrese C, Mangiulli M, Manzari C, Paluscio AM, Caratozzolo MF, Marzano F, Kurelac I, D'Erchia AM, D'Elia D, Licciulli F, Liuni S, Picardi E, Attimonelli M, Gasparre G, Porcelli AM, Pesole G, Sbisa E, and Tullo A. 2013. A platform independent RNA-Seq protocol for the detection of transcriptome complexity. BMC Genomics 14:855. 10.1186/1471-2164-14-855

Caporaso JG, Kuczynski J, Stombaugh J, Bittinger K, Bushman FD, Costello EK, Fierer N, Pena AG, Goodrich JK, Gordon JI, Huttley GA, Kelley ST, Knights D, Koenig JE, Ley RE, Lozupone CA, McDonald D, Muegge BD, Pirrung M, Reeder J, Sevinsky JR, Turnbaugh PJ, Walters WA, Widmann J, Yatsunenko T, Zaneveld J, and Knight R. 2010. QIIME allows analysis of highthroughput community sequencing data. Nat Methods 7:335-336. 10.1038/nmeth.f.303

Chen W, Zhang CK, Cheng YM, Zhang SW, and Zhao HY. 2013. A Comparison of Methods for Clustering 16S rRNA Sequences into OTUs. PLoS One 8. ARTN e70837

10.1371/journal.pone.0070837

Coissac E, Riaz T, and Puillandre N. 2012. Bioinformatic challenges for DNA metabarcoding of plants and animals. Mol Ecol 21:1834-1847. 10.1111/j.1365-294X.2012.05550.x

Comtet T, Sandionigi A, Viard F, and Casiraghi M. 2015. DNA (meta)barcoding of biological invasions: a powerful tool to elucidate invasion processes and help managing aliens. Biological Invasions 17:905-922. DOI 10.1007/s10530-015-0854-y

Cowart DA, Pinheiro M, Mouchel O, Maguer M, Grall J, Mine J, and Arnaud-Haond S. 2015. Metabarcoding is powerful yet still blind: a comparative analysis of morphological and molecular surveys of seagrass communities. PLoS One 10:e0117562. 10.1371/journal.pone.0117562

Cristescu ME. 2014. From barcoding single individuals to metabarcoding biological communities: towards an integrative approach to the study of global biodiversity. Trends Ecol Evol 29:566-571. 10.1016/j.tree.2014.08.001

De Barba M, Miquel C, Boyer F, Mercier C, Rioux D, Coissac E, and Taberlet P. 2014. DNA metabarcoding multiplexing and validation of data accuracy for diet assessment: application to omnivorous diet. Mol Ecol Resour 14:306-323. 10.1111/1755-0998.12188

Deagle BE, Jarman SN, Coissac E, Pompanon F, and Taberlet P. 2014. DNA metabarcoding and the cytochrome c oxidase subunit I marker: not a perfect match. Biol Lett 10. 10.1098/rsbl.2014.0562 
477 Edgar RC. 2004. MUSCLE: multiple sequence alignment with high accuracy and high throughput.

478

479

480

481

482

483

484

485

486

487

488

489

490

491

492

493

494

495

496

497

498

499

500

501

502

503

504

505

506

507

508

509

Nucleic Acids Res 32:1792-1797. 10.1093/nar/gkh340

Edgar RC. 2013. UPARSE: highly accurate OTU sequences from microbial amplicon reads. Nat Methods 10:996-+. 10.1038/Nmeth.2604

Edgar RC, Haas BJ, Clemente JC, Quince C, and Knight R. 2011. UCHIME improves sensitivity and speed of chimera detection. Bioinformatics 27:2194-2200. 10.1093/bioinformatics/btr381

Fantini E, Gianese G, Giuliano G, and Fiore A. 2015. Bacterial metabarcoding by 16S rRNA gene ion torrent amplicon sequencing. Methods Mol Biol 1231:77-90. 10.1007/978-1-4939-1720-4_5

Ficetola GF, Pansu J, Bonin A, Coissac E, Giguet-Covex C, De Barba M, Gielly L, Lopes CM, Boyer F, Pompanon F, Raye G, and Taberlet P. 2015. Replication levels, false presences and the estimation of the presence/absence from eDNA metabarcoding data. Mol Ecol Resour 15:543-556. $10.1111 / 1755-0998.12338$

Finn RD, Clements J, and Eddy SR. 2011. HMMER web server: interactive sequence similarity searching. Nucleic Acids Res 39:W29-37. 10.1093/nar/gkr367

Folmer O, Black M, Hoeh W, Lutz R, and Vrijenhoek R. 1994. DNA primers for amplification of mitochondrial cytochrome c oxidase subunit I from diverse metazoan invertebrates. Mol Mar Biol Biotechnol 3:294-299.

Fonseca VG, Carvalho GR, Nichols B, Quince C, Johnson HF, Neill SP, Lambshead JD, Thomas WK, Power DM, and Creer S. 2014. Metagenetic analysis of patterns of distribution and diversity of marine meiobenthic eukaryotes. Global Ecology and Biogeography 23:1293-1302. Doi 10.1111/Geb.12223

Fonseca VG, Carvalho GR, Sung W, Johnson HF, Power DM, Neill SP, Packer M, Blaxter ML, Lambshead PJ, Thomas WK, and Creer S. 2010. Second-generation environmental sequencing unmasks marine metazoan biodiversity. Nat Commun 1:98. 10.1038/ncomms1095

Geisen S, Laros I, Vizcaino A, Bonkowski M, and de Groot GA. 2015. Not all are free-living: highthroughput DNA metabarcoding reveals a diverse community of protists parasitizing soil metazoa. Mol Ecol. 10.1111/mec.13238

Geml J, Gravendeel B, van der Gaag KJ, Neilen M, Lammers Y, Raes N, Semenova TA, de Knijff P, and Noordeloos ME. 2014. The contribution of DNA metabarcoding to fungal conservation: diversity assessment, habitat partitioning and mapping red-listed fungi in protected coastal Salix repens communities in the Netherlands. PLoS One 9:e99852. 10.1371/journal.pone.0099852

Gibson J, Shokralla S, Porter TM, King I, van Konynenburg S, Janzen DH, Hallwachs W, and Hajibabaei M. 2014. Simultaneous assessment of the macrobiome and microbiome in a bulk sample of 
510

511

512

513

514

515

516

517

518

519

520

521

522

523

524

525

526

527

528

529

530

531

532

533

534

535

536

537

538

539

540

541

542

tropical arthropods through DNA metasystematics. Proc Natl Acad Sci U S A 111:8007-8012. 10.1073/pnas.1406468111

Hajibabaei M. 2012. The golden age of DNA metasystematics. Trends Genet 28:535-537. 10.1016/j.tig.2012.08.001

Hebert PD, Ratnasingham S, and deWaard JR. 2003. Barcoding animal life: cytochrome c oxidase subunit 1 divergences among closely related species. Proc Biol Sci 270 Suppl 1:S96-99. $10.1098 / \mathrm{rsbl} .2003 .0025$

Hoff KJ. 2009. The effect of sequencing errors on metagenomic gene prediction. BMC Genomics 10. Artn 520

10.1186/1471-2164-10-520

Ji Y, Ashton L, Pedley SM, Edwards DP, Tang Y, Nakamura A, Kitching R, Dolman PM, Woodcock P, Edwards FA, Larsen TH, Hsu WW, Benedick S, Hamer KC, Wilcove DS, Bruce C, Wang X, Levi T, Lott M, Emerson BC, and Yu DW. 2013. Reliable, verifiable and efficient monitoring of biodiversity via metabarcoding. Ecol Lett 16:1245-1257. 10.1111/ele.12162

Jost L. 2006. Entropy and diversity. Oikos 113:363-375. DOI 10.1111/j.2006.0030-1299.14714.x

Kajtoch L. 2014. A DNA metabarcoding study of a polyphagous beetle dietary diversity: the utility of barcodes and sequencing techniques. Folia Biol (Krakow) 62:223-234.

Leray M, and Knowlton N. 2015. DNA barcoding and metabarcoding of standardized samples reveal patterns of marine benthic diversity. Proc Natl Acad Sci U S A 112:2076-2081. 10.1073/pnas.1424997112

Matsen FA, Kodner RB, and Armbrust EV. 2010. pplacer: linear time maximum-likelihood and Bayesian phylogenetic placement of sequences onto a fixed reference tree. BMC Bioinformatics 11. Artn 538

10.1186/1471-2105-11-538

Mollot G, Duyck PF, Lefeuvre P, Lescourret F, Martin JF, Piry S, Canard E, and Tixier P. 2014. Cover cropping alters the diet of arthropods in a banana plantation: a metabarcoding approach. PLoS One 9:e93740. 10.1371/journal.pone.0093740

Nanjappa D, Audic S, Romac S, Kooistra WH, and Zingone A. 2014. Assessment of species diversity and distribution of an ancient diatom lineage using a DNA metabarcoding approach. PLoS One 9:e103810. 10.1371/journal.pone.0103810

Op De Beeck M, Lievens B, Busschaert P, Declerck S, Vangronsveld J, and Colpaert JV. 2014. Comparison and validation of some ITS primer pairs useful for fungal metabarcoding studies. PLoS One 9:e97629. 10.1371/journal.pone.0097629 
543 Pawluczyk M, Weiss J, Links MG, Egana Aranguren M, Wilkinson MD, and Egea-Cortines M. 2015.

544

545

546

547

548

549

550

551

552

553

554

555

556

557

558

559

560

561

562

563

564

565

566

567

568

569

570

571

572

573

574

575

Quantitative evaluation of bias in PCR amplification and next-generation sequencing derived from metabarcoding samples. Anal Bioanal Chem 407:1841-1848. 10.1007/s00216-014-8435-y

Pinol J, Mir G, Gomez-Polo P, and Agusti N. 2014. Universal and blocking primer mismatches limit the use of high-throughput DNA sequencing for the quantitative metabarcoding of arthropods. $\mathrm{Mol}$ Ecol Resour. 10.1111/1755-0998.12355

Quemere E, Hibert F, Miquel C, Lhuillier E, Rasolondraibe E, Champeau J, Rabarivola C, Nusbaumer L, Chatelain C, Gautier L, Ranirison P, Crouau-Roy B, Taberlet P, and Chikhi L. 2013. A DNA metabarcoding study of a primate dietary diversity and plasticity across its entire fragmented range. PLoS One 8:e58971. 10.1371/journal.pone.0058971

Quince C, Lanzen A, Davenport RJ, and Turnbaugh PJ. 2011. Removing noise from pyrosequenced amplicons. BMC Bioinformatics 12:38. 10.1186/1471-2105-12-38

Schloss PD, Gevers D, and Westcott SL. 2011. Reducing the effects of PCR amplification and sequencing artifacts on 16S rRNA-based studies. PLoS One 6:e27310. 10.1371/journal.pone.0027310

Shokralla S, Spall JL, Gibson JF, and Hajibabaei M. 2012. Next-generation sequencing technologies for environmental DNA research. Mol Ecol 21:1794-1805. 10.1111/j.1365-294X.2012.05538.x

Singh J, Behal A, Singla N, Joshi A, Birbian N, Singh S, Bali V, and Batra N. 2009. Metagenomics: Concept, methodology, ecological inference and recent advances. Biotechnol J 4:480-494. 10.1002/biot.200800201

Sogin ML, Morrison HG, Huber JA, Mark Welch D, Huse SM, Neal PR, Arrieta JM, and Herndl GJ. 2006. Microbial diversity in the deep sea and the underexplored "rare biosphere". Proc Natl Acad Sci U S A 103:12115-12120. 10.1073/pnas.0605127103

Srivathsan A, Sha JC, Vogler AP, and Meier R. 2015. Comparing the effectiveness of metagenomics and metabarcoding for diet analysis of a leaf-feeding monkey (Pygathrix nemaeus). Mol Ecol Resour 15:250-261. 10.1111/1755-0998.12302

Stamatakis A. 2014. RAxML version 8: a tool for phylogenetic analysis and post-analysis of large phylogenies. Bioinformatics 30:1312-1313. 10.1093/bioinformatics/btu033

Taberlet P, Coissac E, Pompanon F, Brochmann C, and Willerslev E. 2012. Towards next-generation biodiversity assessment using DNA metabarcoding. Mol Ecol 21:2045-2050. 10.1111/j.1365294X.2012.05470.x

Thomas AC, Deagle, B. E., Eveson, J. P., Harsch, C. H. and Trites, A. W. . 2015. Quantitative DNA metabarcoding: improved estimates of species proportional biomass using correction factors derivedfrom control material. Mol Ecol Resour. 10.1111/1755-0998.12490

PeerJ reviewing PDF | (2017:08:20240:2:0:NEW 2 May 2018) 
576 Xu C, Dong WP, Shi S, Cheng T, Li CH, Liu YL, Wu P, Wu HK, Gao P, and Zhou SL. 2015.

577 Accelerating plant DNA barcode reference library construction using herbarium specimens:

578 improved experimental techniques. Mol Ecol Resour 15:1366-1374. 10.1111/1755-0998.12413

579 Zhan A, Bailey SA, Heath DD, and Macisaac HJ. 2014. Performance comparison of genetic markers for 580 high-throughput sequencing-based biodiversity assessment in complex communities. Mol Ecol

$581 \quad$ Resour 14:1049-1059. 10.1111/1755-0998.12254

582 


\section{Figure 1}

Bioinformatics analysis pipelines overview.

The same reference database is used by all methods to assign each sequence to a known taxon name. RDP and BLAST classifiers take as input the output of OTU picking methods (Usearch, Uclust, Blast). HSTA, Usearch-Ref and Uclust-Ref use the denoised sequences as direct input. The Bayes Factor $(B F)$ equation is a logical representation of that implemented in HSTA (see OTU picking and Taxonomic Assignment section, letter c.)

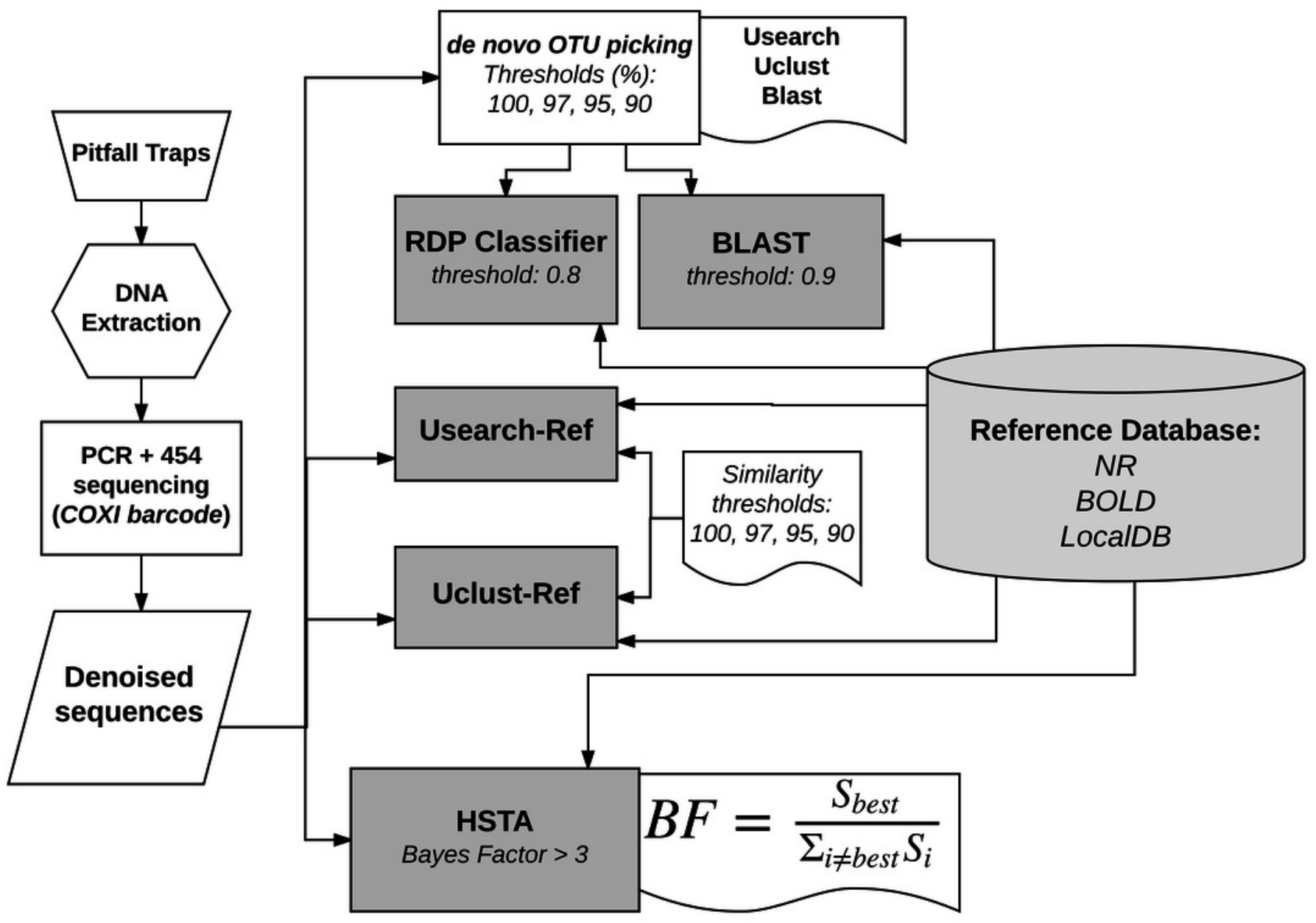


Figure 2

Order level taxonomic assignments in MPE5 sample.

(A) Forward strand or 5' coxl, (B) reverse strand or 3' coxl. Each color corresponds to a taxonomic classifier (HSTA, RDP, BLAST) or a reference-based assigner algorithm (UsearchRef, Uclust-Ref). The symbols indicate either the combination of an OTU picking method with a classifier or the classifier/algorithm used for direct taxonomic assignment. X-axis corresponds to the similarity thresholds used in OTU picking or with the direct assignments in HSTA and reference-based algorithms.

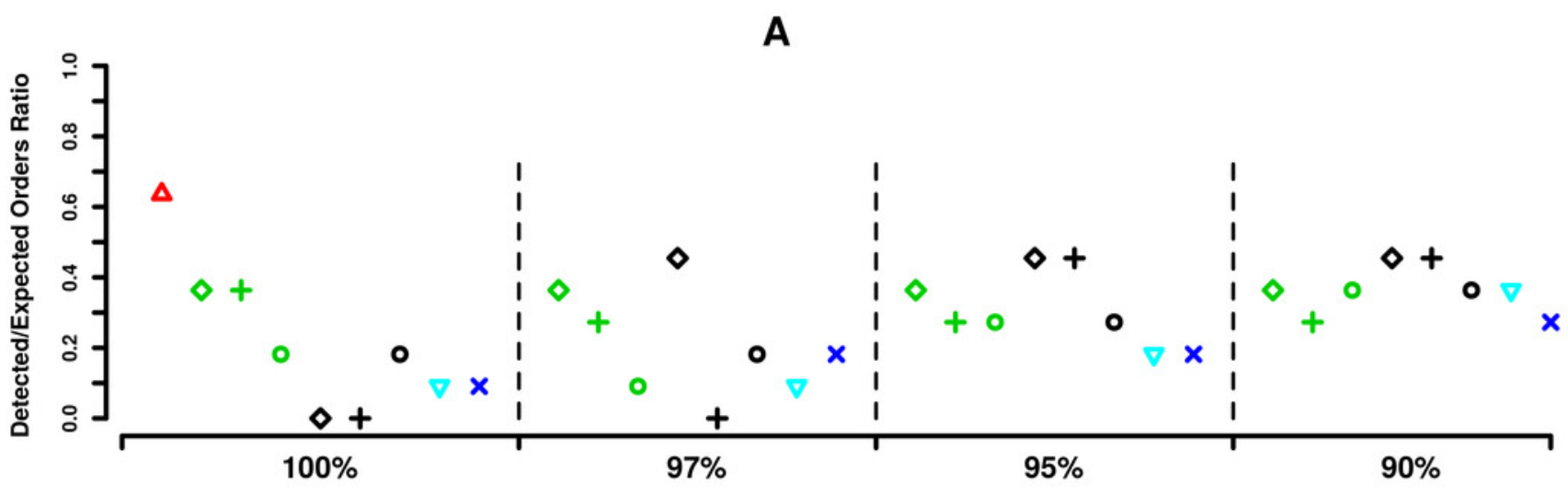

Similarity Threshold

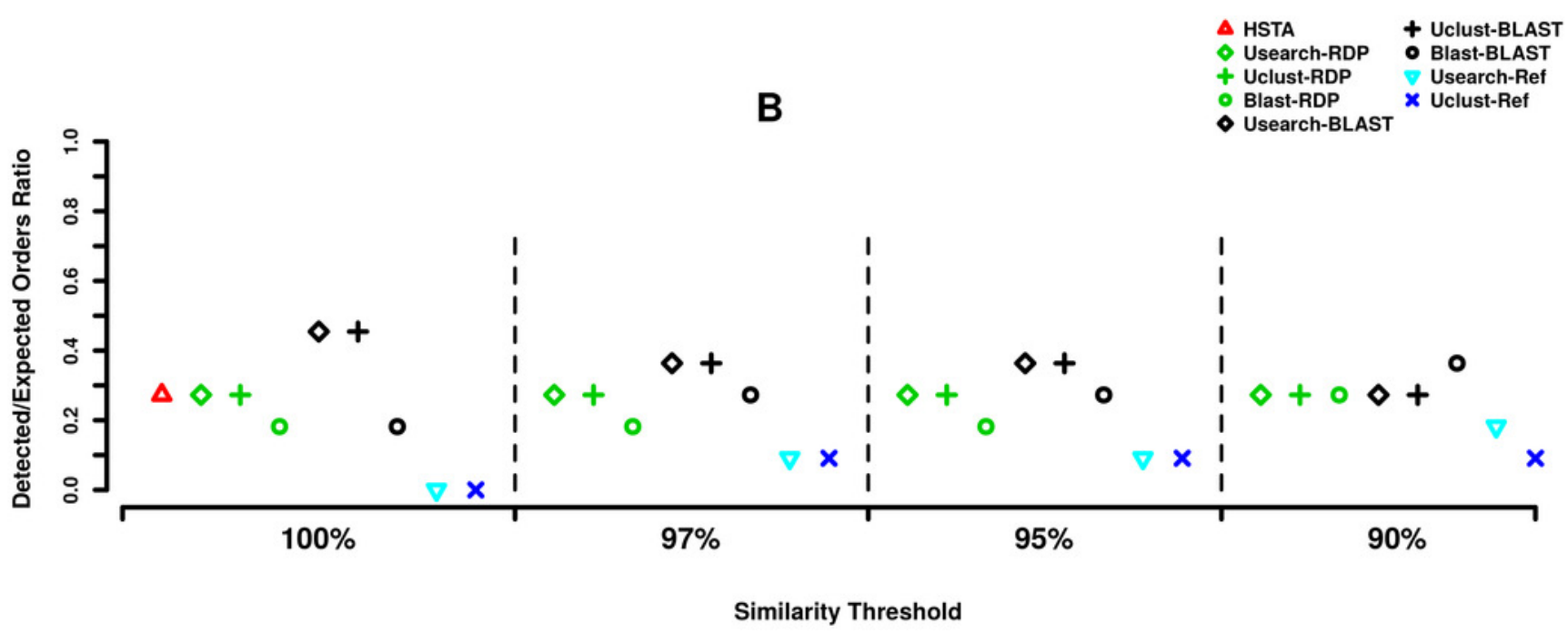


Figure 3

MPE5 taxonomic assignments plots at species level.

(A) Forward strand or 5' coxl, (B) reverse strand or 3' coxl. Each color corresponds to a taxonomic classifier (HSTA, RDP, BLAST) or a reference-based assigner algorithm (UsearchRef, Uclust-Ref). The symbols indicate either the combination of an OTU picking method with a classifier or the classifier/algorithm used for direct taxonomic assignment. X-axis corresponds to the similarity thresholds used in OTU picking or with the direct assignment in HSTA and reference-based algorithms.

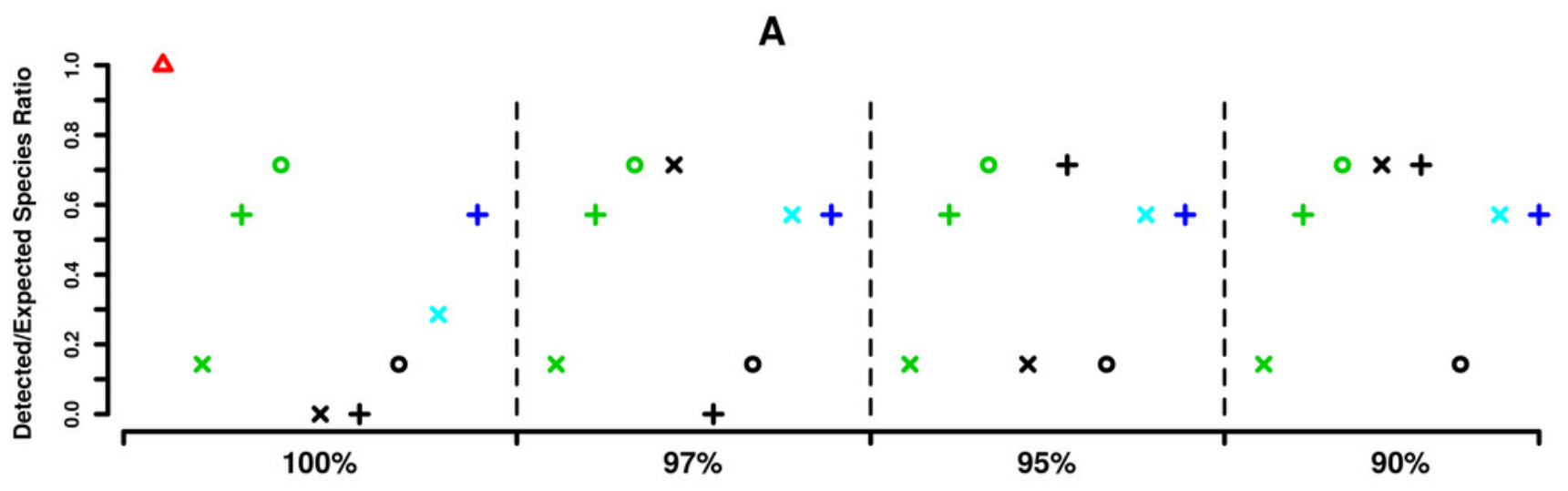

Similarity Threshold

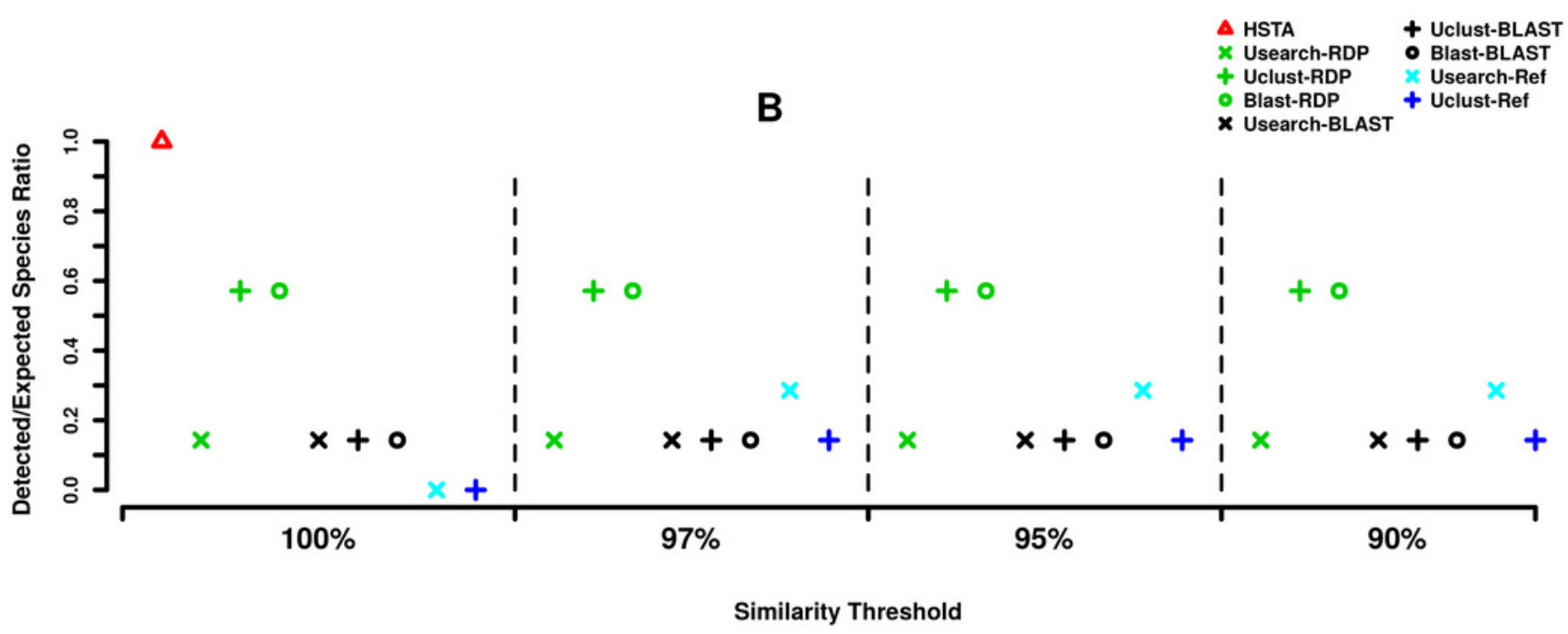




\section{Table $\mathbf{1}$ (on next page)}

Taxonomically identified organisms in MPE5 sample and their corresponding biomass.

MPE4 simply contains the same organisms at equal biomass. 


\begin{tabular}{|c|c|c|c|}
\hline Taxonomic Group & $\begin{array}{c}\text { Total Biomass } \\
\text { (g) }\end{array}$ & Species Name & $\begin{array}{c}\text { Biomass } \\
\text { of single } \\
\text { organism } \\
\text { (g) }\end{array}$ \\
\hline \multirow[t]{7}{*}{$\begin{array}{c}\text { Coleoptera (family: } \\
\text { Carabidae) }\end{array}$} & 22.89 & $\begin{array}{c}\text { Carabus (Tomocarabus) } \\
\text { convexus dilatatus }^{\#}\end{array}$ & 12.94 \\
\hline & & $\begin{array}{c}\text { Carabus (Chaetocarabus) } \\
\text { lefebvrei bayardi }\end{array}$ & 4.85 \\
\hline & & Calathus fracasii & 0.58 \\
\hline & & Abax parallelepipedus & 0.29 \\
\hline & & Laemostenus latialis ${ }^{\#}$ & 0.23 \\
\hline & & Pterostichus micans & 0.18 \\
\hline & & Calathus montivagus & 0.07 \\
\hline Diptera & 4.12 & & \\
\hline Orthoptera & 2.44 & & \\
\hline Blattodea & 1.02 & & \\
\hline Myriapoda* & 0.82 & & \\
\hline Isopoda & 0.7 & & \\
\hline Arachnida* & 0.64 & & \\
\hline Scorpiones & 0.63 & & \\
\hline Hymenoptera & 0.21 & & \\
\hline Lepidoptera & 0.1 & & \\
\hline Collembola & 0.02 & & \\
\hline
\end{tabular}

1 *Class taxonomy rank. "Laemostenus latialis = LL1 and Carabus (Tomocarabus) convexus 2 dilatatus $=\mathrm{CC} 1$ 


\section{Table 2 (on next page)}

Sites diversity in sequence reads predicted by sites diversity in references and by GC content.

The results are reported for sequences assigned to Coleoptera order over the $5^{\prime}$ and $3^{\prime}$ end of coxl barcode region. 


\begin{tabular}{|c|c|c|c|c|c|c|}
\hline & \multicolumn{3}{|c|}{$\begin{array}{c}5^{\prime} \text { coxI barcode } \\
\text { analyzed sites: } 1-400\end{array}$} & \multicolumn{3}{|c|}{$\begin{array}{c}\text { 3' coxI barcode } \\
\text { analyzed sites: } 200 \text {-end }\end{array}$} \\
\hline & GCref & Dref & Residuals & GCref & Dref & Residuals \\
\hline $\begin{array}{l}\text { Degree of } \\
\text { freedom }\end{array}$ & 1 & 1 & 562 & 1 & 1 & 562 \\
\hline $\begin{array}{l}\text { Sum of } \\
\text { squares }\end{array}$ & 4.518 & 58.766 & 123.808 & 37.999 & 50.025 & 135.407 \\
\hline $\begin{array}{l}\text { Mean } \\
\text { square }\end{array}$ & 4.518 & 58.766 & 0.220 & 37.999 & 50.025 & 0.241 \\
\hline F-value & 20.507 & 266.754 & - & 157.71 & 207.63 & - \\
\hline $\begin{array}{c}\text { Explained } \\
\text { Variance } \\
(\%)\end{array}$ & 2,41 & 31,41 & 66,17 & 17 & 22,3 & 66,17 \\
\hline $\operatorname{Pr}(>F)$ & $\begin{array}{c}7.257 \mathrm{e}-06 \\
* * *\end{array}$ & $\begin{array}{c}<2.2 \mathrm{e}-16 \\
* * *\end{array}$ & - & $\begin{array}{c}<2.2 \mathrm{e}-16 \\
* * *\end{array}$ & $\begin{array}{c}<2.2 \mathrm{e}-16 \\
* * *\end{array}$ & - \\
\hline
\end{tabular}

1 GCref and Dref are the GC content and sites diversity of reference sequences respectively.

2 Significance codes: 0 ' $* * *$ ' $0.001^{\prime} * *$ ' $0.01^{\prime \prime} *$ ' $0.05^{\prime}$ ' 


\section{Table 3(on next page)}

Universal primers amplification bias analysis results of MPE5 and MPE4 samples.

All the reported values refer to the ratio CC1/LL1 of qPCR intensity signal for DNA extract, PCR products and library preparation categories while 5' and 3' counts correspond to the number of assigned reads for CC1 and LL1 species obtained from HSTA pipeline. LL1 = Laemostenus latialis, $\mathrm{CC1}$ = Carabus (Tomocarabus) convexus dilatatus. 


\begin{tabular}{|c|c|c|c|c|c|c|}
\hline & & \multicolumn{3}{|c|}{ qPCR signal intensity } & \multicolumn{2}{|c|}{ HSTA assigned reads } \\
\hline $\begin{array}{c}\text { Sampl } \\
\text { e }\end{array}$ & $\begin{array}{c}\text { Biomas } \\
\text { s }\end{array}$ & $\begin{array}{c}\text { DNA } \\
\text { extract }\end{array}$ & $\begin{array}{c}\text { PCR } \\
\text { products }\end{array}$ & $\begin{array}{c}\text { Library } \\
\text { Preparation }\end{array}$ & $\begin{array}{c}\text { Count at } \\
\text {, }\end{array}$ & $\begin{array}{c}\text { Count at } \\
3\end{array}$ \\
\hline MPE5 & 56.26 & 1508.46 & 620.31 & 122.34 & 231.35 & 103.55 \\
\hline MPE4 & 1 & 53.92 & 163.76 & 19.59 & 1.83 & 1.29 \\
\hline
\end{tabular}

